\title{
SCALING OF THIRD-ORDER QUADRUPOLE ABERRATIONS WITH FRINGE FIELD EXTENSION*
}

\author{
M. Venturini ${ }^{\dagger}$ \\ Physics Department, University of Maryland, College Park, Maryland 20742
}

\section{Abstract}

We present a simplified analytical model that shows how fringe field aberrations depend on a quadrupole magnet aperture. It is found that for a fixed magnet length and focal length the fringe field aberrations are smaller if the magnet aperture is larger (i.e. if the fringes are more extended).

\section{INTRODUCTION}

In spite of the progress made in understanding the effects of magnet fringe fields there still seems to be some confusion about the way the aberrations scale with the fringe extension. A popular belief in the accelerator physics community is that the fringe extension should be contained as much as possible. Although there may be some good reasons for that belief, limiting the strength of aberrations is not one of them. A source for this misconception may have been the fact that the intrinsic aberrations associated with the fringes increase as the length of a magnet decreases. That is, given for instance two quadrupoles with the same focal length and same aperture, the aberrations are larger for the magnet with shorter length. Short magnets are associated with relatively more extended fringe fields and this fact may have generated the wrong perception that the more extended the fringes are the higher the aberrations. In fact the opposite turns out to be true. A clarification of this issue is desirable because short-length, large-aperture magnets are increasingly being used in a number of applications. In this paper we calculate and compare the third order aberrations associated with the fringes of quadrupole magnets having the same length and same integrated on-axis gradient but different apertures (and therefore different extension of the fringes). The calculation is done for a simplified 1D model for which one can calculate the third order aberrations associated with the fringes analytically. The approximated analytical formulas are compared with an exact numerical computation carried out using MARYLIE [1]. We find that the aberrations decrease exponentially with the square root of the magnet aperture.

\section{TRANSFER MAP COMPUTATION}

A convenient way to represent the dynamics of a charged particle through a magnet is to write the associated transfer map in the Lie form [2]:

$$
\mathcal{M}=\cdots \exp \left(: f_{5}:\right) \exp \left(: f_{4}:\right) \exp \left(: f_{3}:\right) \mathcal{M}_{2} .
$$

\footnotetext{
* Work supported by the U.S. Department of Energy.

†venturin@physics.umd.edu
}

The linear content of the dynamics is described by $\mathcal{M}_{2}$ while the nonlinear part is represented by the Lie generators $f_{n}$. The $f_{n}$ are homogeneous polynomials of order $n$ in the dynamical variables and $f_{n}$ : are the Lie operators associated with $f_{n}$, i.e. : $f_{n}: g=[f, g]$ with $[\cdot, \cdot]$ being the Poisson brackets.

A map can be calculated by solving the canonical equation $\mathcal{M}^{\prime}=\mathcal{M}:-H$ :. In particular the linear part of the map is a solution of

$$
\mathcal{M}_{2}^{\prime}=\mathcal{M}_{2}:-H_{2}: \text {. }
$$

Here $H_{2}$ denotes the quadratic part of the Hamiltonain, which we assume can be written as a series $\mathrm{H}=\mathrm{H}_{2}+$ $H_{3}+H_{4}+\cdots$ with $H_{3}$ being the cubic part, $H_{4}$ the quartic part etc. . It can be shown that $H_{2}$ contributes to $\mathcal{M}_{2}$ and all the $f_{n} ; H_{3}$ contributes to the $f_{n}$ with $n=3$ and higher and so on. For the case considered in this paper $H_{3}=0$, and therefore the first nonlinear generator is $f_{4}$. It can be shown [2] that:

$$
f_{4}=-\int_{z_{i}}^{z_{f}} \mathcal{M}_{2}^{z_{i} \rightarrow z} H_{4}(z) d z
$$

where with $\mathcal{M}_{2}^{z_{i} \rightarrow z}$ we indicate the solution of (2) from $z=z_{i}$ (some point before the magnet entry) to $z_{f}$ (some point past the magnet exit). The generator $f_{4}$ contributes to the third and higher order aberrations that appear in a Taylor representation of the transfer map. We now consider a 1-D model of charged particle dynamics in a quadrupole magnet described by the Hamiltonian:

$$
H=\frac{p_{x}^{2}}{2}+\frac{x^{2}}{2} k(z)+\frac{p_{x}^{4}}{8}+\frac{x^{4}}{12} k^{\prime \prime}(z)+\frac{x^{3} p_{x}}{4} k^{\prime}(z) .
$$

The focusing function $k(x)$ equals the magnetic rigidity times the on-axis gradient: $k(x)=\left(q / p^{o}\right) \mathcal{G}(z)$. Apart from the reduced dimensionality and omission of chromatic terms the Hamiltonian above is exact through $4^{\text {th }}$ order. By indicating with $m_{i j}(z)$ the matrix representation of the linear part of the map, the $4^{t h}$ order Lie generator can be written as $f_{4}=f_{4}^{g e o m}+f_{4}^{d y n}$ where:

$$
f_{4}^{\text {geom }}=-\frac{1}{8} \int_{z_{i}}^{z_{f}}\left(m_{21} x+m_{22} p_{x}\right)^{4} d z .
$$

and

$$
f_{4}^{d y n}=-\frac{1}{4} \int_{z_{i}}^{z_{f}}\left[\frac{k^{\prime \prime}(z)}{3} \mathcal{M}_{2} x^{4}+k^{\prime}(z) \mathcal{M}_{2} p_{x} x^{3}\right] d z .
$$




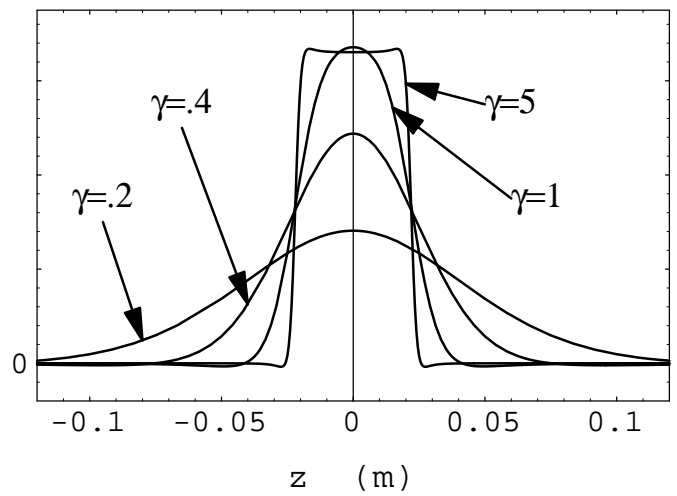

Figure 1: Profiles of the focusing function (9) for different values of the aspect ratio $\gamma=l / R$; the quadrupole semilength is $l=.022 \mathrm{~m}$. The integrals of the various curves are the same.

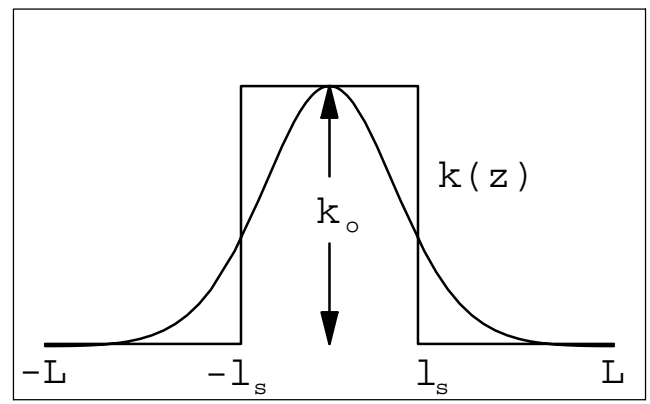

Figure 2: Approximation of the focusing function with a stepwise function.

After an integration by parts, use of Eq. (2) and the relationship

$$
: H_{2}: x^{4}=\left[H_{2}, x^{4}\right]=\left[\frac{1}{2} p_{x}^{2}+\frac{1}{2} k(z) x^{2}, x^{4}\right]=-4 x^{3} p_{x},
$$

finally yield

$$
\begin{aligned}
& f_{4}^{d y n}=\frac{1}{12} \int_{z_{i}}^{z_{f}} k^{\prime}(z) \mathcal{M}_{2} x^{3} p_{x} d z \\
= & \frac{1}{12} \int_{z_{i}}^{z_{f}} k^{\prime}(z)\left(m_{11} x+m_{12} p_{x}\right)^{3}\left(m_{21} x+m_{22} p_{x}\right) d z .
\end{aligned}
$$

Because $f_{4}^{\text {geom }}$ does not depend on the quadrupole fields, in the following we consider $f_{4}^{d y n}$ only. Our goal is now to evaluate the integral (8) for a specific choice of $k(z)$.

\section{COMPUTING THE ABERRATIONS}

Consider a family of quadrupoles with focusing function

$$
k(z, R)=g_{0}[G(z+l)-G(z-l)],
$$

and

$$
G(t)=\frac{1}{2} \frac{R^{4} t}{\left(R^{2}+t^{2}\right)^{\frac{5}{2}}}+\frac{1}{3} \frac{t\left(3 R^{2}+2 t^{2}\right)}{\left(R^{2}+t^{2}\right)^{\frac{3}{2}}}
$$

where $R$ and $l$ are the magnet aperture and semilength. A focusing function of the form (9) is typical of ironfree quadrupole magnets like those used in the Electron Ring under construction at the University of Maryland [3]. Here we are interested in comparing quadrupole magnets that have the same length $2 l$ but different aperture $2 R$. The function (9) is defined in such a way that the $z$-integral (and therefore the magnet focal length in first approximation) does not depend on the quadrupole aperture: $\int k(z, R) d z=8 g_{0} l / 3$. Physically this is achieved by powering the magnets in a way dependent on their aperture. The profiles for 4 different choices of the apertures are shown in Fig 1. The 'aspect ratio' $\gamma$ is defined as the ratio between the magnet length and aperture: $\gamma=l / R$. Notice that $\gamma \rightarrow \infty$ (i.e. $R \rightarrow 0$ ) represents the hard edge limit. In order to compute the integral (8) we first need to find the linear part of the map. We do the calculation in an approximated manner by assuming that we can represent $k(z)$ as a stepwise function, (see Fig. 2). Two parameters need to be specified: the length $l_{s}$ and the peak value $k_{o}$ of $k_{s t e p}(z)$. A constraint we impose is that the integral of $k(z)$ equals that of $k_{\text {step }}(z)$. Therefore we need to specify either $k_{o}$ or $l_{s}$, for any given value of the aspect ratio. We can expect that as the aspect ratio decreases and the extension of the fringes increases, $l_{s}$ should become larger and $k_{o}$ smaller. A possible choice is to use the standard definition of a magnet effective length and write: $k_{o}=k(z=0, R)$. Instead we use a slightly modified expression

$$
k_{o}=k\left(z=0, R\left(1+\gamma \gamma_{1}^{-1}\right)\right)
$$

to allow for the presence of a free parameter $\gamma_{1}$ to be determined later. The length $l_{s}$ is then determined by requiring that $l_{s} k_{o}$ is the same as the integrated gradient. With these assumptions the elements $m_{i j}$ of the transfer matrix from $z=-L$ to $z \in\left[-l_{s}, l_{s}\right]$ are $m_{11}=\cos \omega\left(z+l_{s}\right)$, $m_{21}=-\omega \sin \omega\left(z+l_{s}\right)$, etc., with $\omega=\sqrt{k_{o}}$. As a further approximation we assume that these expressions for the elements of the transfer matrix can be extended to $|z|>l_{s}$. This is plausible because the error we introduce in this way is small as $k(z)$ decays rapidly for $|z|>l_{s}$. For the same reason we can approximate $z_{i}=-L \simeq-\infty$ and $z_{f}=L \simeq \infty$ in the the integral (8). The Lie generator for the third order aberrations for the motion in the $x$ plane consists of 5 monomials:

$$
\begin{aligned}
f_{4}^{d y n}= & x^{4} f_{x^{4}}^{d y n}+x^{3} p_{x} f_{x^{3} p_{x}}^{d y n}+x^{2} p_{x}^{2} f_{x^{2} p_{x}^{2}}^{d y n} \\
& +x p_{x}^{3} f_{x p_{x}^{3}}^{d y n}+p_{x}^{4} f_{p_{x}^{4}}^{d y n} .
\end{aligned}
$$

Let us focus on $f_{x^{4}}^{d y n}$. By using the approximations we have indicated above we have:

$f_{x^{4}}^{d y n} \simeq \frac{-1}{12} \int_{-\infty}^{\infty} k^{\prime}(z)\left[\cos \omega\left(z+l_{s}\right)\right]^{3}\left[\omega \sin \omega\left(z+l_{s}\right)\right] d z$.

This integral can be expressed in terms of the modified Bessel functions $K_{0}$ and $K_{1}$. Having introduced the defin- 


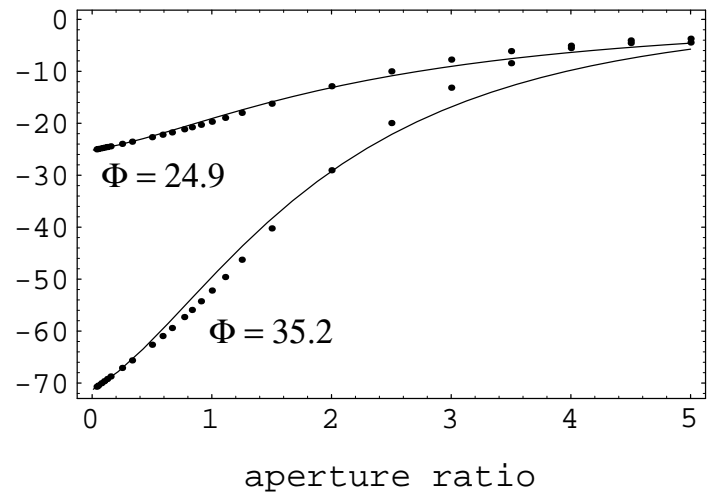

Figure 3: Lie generator $f_{x^{4}}^{d y n}$ (in units of $m^{-4}$ ) as a function of the aperture ratio $R / l$ for various values of the phase advance $\Phi$ (expressed in deg). The solid lines represent Eq. (15), the dots are from the MARYLIE calculation.

ition of the function $I(\Omega)$

$$
\begin{aligned}
I(\Omega) \equiv & \int_{-\infty}^{\infty} k^{\prime}(z) \sin (\Omega z) d z=\frac{-4 l}{3} g_{o} \Omega R \sin (\Omega l) \times \\
& \left\{\left[4+(\Omega R)^{2}\right] K_{1}(\Omega R)+2(\Omega R) K_{0}(\Omega R)\right\},
\end{aligned}
$$

we find

$$
f_{x^{4}}^{d y n} \simeq \frac{\omega}{48}\left[I(2 \omega) \cos \Phi+\frac{1}{2} I(4 \omega) \cos 2 \Phi\right],
$$

where $\Phi=2 \omega l_{s}$ is the phase advance between $z=-l_{s}$ and $z=l_{s}$. In a similar way we can calculate

$$
\begin{aligned}
f_{x^{3} p_{x}}^{d y n} \simeq & -\frac{I(2 \omega)}{24}\left[\sin \Phi+2\left(L-l_{s}\right) \omega \cos \Phi\right] \\
& -\frac{I(4 \omega)}{24}\left[\sin 2 \Phi+\left(L-l_{s}\right) \omega \cos 2 \Phi\right],
\end{aligned}
$$

as well as the remaining coefficients in (12) which we not report here. They all contain terms proportional to either $I(2 \omega)$ or $I(4 \omega)$. From the expression above one can recover the aberrations in the hard edge limit $R \rightarrow 0$ (with $l$ kept fixed). We have

$$
\lim _{R \rightarrow 0} I(\Omega)=-\omega^{2} \sin (\Omega l)
$$

and hence for example:

$$
f_{x^{4}}^{d y n}=-\frac{\omega^{3}}{48}\left[\sin 2 \Phi+\frac{1}{2} \sin 4 \Phi\right] .
$$

In the hard edge limit the quantities $\omega$ and $\Phi$ appearing in the expressions above are related by $2 \omega l=\Phi$ because $l_{s}=l$. In the hard edge limit these expressions are exact and are consistent with the analytical formulas found in the literature. The limiting form in the infinitely soft limit can also be written using

$$
\begin{aligned}
\lim _{R \rightarrow \infty} I(\Omega)= & -\frac{4 l}{3} g_{o} \sqrt{\frac{\pi}{2}} \sin (\Omega l) \exp (-\Omega R) \times \\
& {\left[4(\Omega R)^{\frac{1}{2}}+2(\Omega R)^{\frac{3}{2}}+(\Omega R)^{\frac{5}{2}}\right] }
\end{aligned}
$$

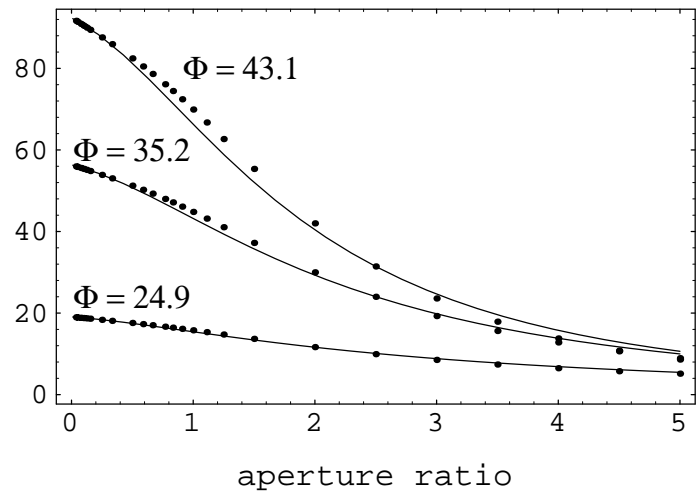

Figure 4: Lie generator $f_{x^{3} p_{x}}^{d y n}$ (in units of $m^{-3}$ ) as a function of the aperture ratio $R / l$ for various values of the phase advance $\Phi$ (expressed in deg). The solid lines represent Eq. (15) the dots are from the MARYLIE calculation.

Notice that the aberrations decrease exponentially with $\Omega R$. For large values of $\mathrm{R}$ we have $\omega \propto 1 / \sqrt{R}$ and therefore $I(2 \omega) \propto \exp (-\sqrt{R} \times$ const $)$. The limiting expression (19) deviates less than a few percents from the exact expression (14) for $\Omega R \geq 2$. The Lie generators $f_{x^{4}}^{d y n}$ an $f_{x^{3} p_{x}}^{d y n}$ as functions of the magnet aperture ratio $(=R / l$, i.e. the inverse of the aspect ratio $\gamma$ ) are shown in Figs. 3 and 4. The solid lines are plots of the analytical formulas for various values of the phase advance $\Phi$ [corresponding to various choices of the constant $g_{o}$ in the expression (9) for $k(z)$ ]. Comparison is made with an exact numerical calculation of the transfer map carried out with MARYLIE (dots). The best agreement is obtained with a value $\gamma_{1}=1 / 1.17$ for the free paramerer $\gamma_{1}$. Notice how the qualitative dependence of the generators on the aperture ratio is well described by the analytical formulas.

\section{CONCLUSION}

The conclusion of this paper is that for the kind of quadrupole magnets considered here less extended fringes carry larger aberrations. The integral (6) defining the strength of the aberrations depends on two opposing factors: the size of the fringe field region (i.e. the interval in $z$ in which the integral is non vanishing) and the derivatives of the focusing function. As a quadrupole magnet aperture decreases the fringe extension decreases but the derivative of the focusing function increases, and between the two the latter prevails.

\section{REFERENCES}

[1] A. Dragt, Release of MARYLIE 3.0, these proceedings.

[2] A. Dragt, Lie Methods for Nonlinear Dynamics with Applications to Accelerator Physics, University of Maryland Physics Department Report (1999).

[3] M. Reiser et al., The Maryland Electron Ring for Investigating Space-Charge Dominated Beams in a Circular FODO System, These Proceedings. 